\title{
Tsafon
}

Revue d'études juives du Nord

$76 \mid 2018$

Expressions yiddish de la nature

\section{«Anno Domini 1933 ». À propos du recueil La Parole des muets de Gertrud Kolmar}

Helmut Pillau

\section{(2) OpenEdition}

1 Journals

Édition électronique

URL : https://journals.openedition.org/tsafon/1423

DOI : $10.4000 /$ tsafon. 1423

ISSN : 2609-6420

Éditeur

Association Jean-Marie Delmaire

Édition imprimée

Date de publication : 1 décembre 2018

Pagination : 143-155

ISSN : 1149-6630

\section{Référence électronique}

Helmut Pillau, « «Anno Domini 1933 ». À propos du recueil La Parole des muets de Gertrud Kolmar »,

Tsafon [En ligne], 76 | 2018, mis en ligne le 30 juin 2019, consulté le 26 juin 2021. URL : http://

journals.openedition.org/tsafon/1423 ; DOI : https://doi.org/10.4000/tsafon.1423

Tsafon. Revues d'études juives du Nord 


\title{
Varia : Littérature
}

\section{«Anno Domini $1933 »$}

\author{
À propos du recueil La Parole des muets de Gertrud Kolmar
}

\section{Helmut Pillau*}

(1)

Lorsque l'on aborde de nos jours le poème «Anno domini 1933 » du recueil Das Wort der Stummen [La Parole des muets] de Gertrud Kolmar, le titre même a quelque chose de déconcertant. 1933, cette année funeste dans l'histoire de l'Allemagne est placée sous le signe de « l'Année du Seigneur»! Dans ce texte rédigé le 16 octobre $1933^{1}$, la poétesse s'insurge contre la collaboration fatale qui s'amorçait déjà entre des pans entiers de l'Église protestante et le régime nazi. En effet, peu de temps auparavant, le 27 septembre 1933, le pasteur Ludwig Müller, appartenant à la mouvance des "Chrétiens allemands", avait été élu «Évêque du Reich» [«Reichsbischof»] lors du synode national qui

\footnotetext{
* Helmut Pillau a enseigné la littérature comparée à la Johannes Gutenberg Universität de Mayence. L'article reprend le texte de sa conférence tenue le 9 novembre 2017 dans le cadre d'une commémoration du pogrome de la nuit du 9 novembre 1938 [«Reichspogromnacht»] organisée par les Églises protestante et catholique et par le forum «Kultur und Politik» de la ville de Heidesheim sur le Rhin. Le texte allemand est consultable en ligne : "'Anno Domini 1933'. Zu dem Gedichtzyklus 'Das Wort der Stummen' von Gertrud Kolmar», http:/www.helmutpillau.com/2017/12/28/annodomini-1933/. Texte traduit de l'allemand par Andrée Lerousseau.

${ }^{1}$ Gertrud Kolmar, Das lyrische Werk, Anhang und Kommentar, hrsg. von Regina Nörtmann, Göttingen, Wallstein-Verlag, 2010 (2. A.), p. 264.
} 
s'était tenu à Wittemberg. Sur le ton du sarcasme, Gertrud Kolmar dénonce énergiquement, à la fin du poème, la symbiose affirmée sous serment entre le christianisme et le national-socialisme : « ̀̀ coups de botte et de matraque / Dans le troisième Reich chrétien-germanique $»^{2}$. «'L'Occident chrétien' fait tomber le masque! » ${ }^{3}$, serait-on tenté de s'exclamer à la lecture de ces paroles lourdes d'amertume.

Outre la rapidité avec laquelle cette Juive allemande réagit à l'événement, on est surpris par la teneur manifestement politique du recueil, rien dans ses écrits antérieurs ne laissant présager un tel engagement.

Son nom de plume, «Kolmar», est un pseudonyme. Officiellement, elle s'appelait « Chodziesner », patronyme dérivé du nom d'une circonscription de la province prussienne de Posnanie dont la famille de son père était originaire. Plus tard, la ville fut rebaptisée «Kolmar ${ }^{4}$, d'où le choix de la poétesse de publier sous ce nom d'emprunt. L'utilisation d'un pseudonyme étant interdite aux Juifs sous le Troisième Reich, elle dut, en tant qu'auteure, avoir de nouveau recours à son patronyme précédé de son prénom auquel elle fut contrainte d'ajouter «Sarah». Elle naquit en 1894 à Berlin où son père s'était installé et où il devint un avocat de renom. Comme cela était courant pour nombre de Juifs assimilés, les liens de son entourage avec le judaïsme, en particulier en matière de religion, s'étaient amenuisés considérablement au fil du temps.

À la différence des autres membres de la famille, Gertrud Kolmar, qui se sentait parfaitement chez elle dans la culture allemande, s'intéressa toutefois de bonne heure au judaïsme, mais ne s'identifia à celui-ci qu'après 1933, tout en demeurant en marge de toute orthodoxie. Elle écrivait des poèmes depuis sa jeunesse, sans se préoccuper outre mesure de leur publication et rares étaient ceux qui estimaient ses écrits à leur juste valeur. Aujourd'hui, son nom est immédiatement et spontanément

\footnotetext{
${ }^{2}$ Gertrud Kolmar, Das lyrische Werk. Gedichte 1927-1937, hrsg. von Regina Nörtmann, Göttingen, Wallstein-Verlag, 2010 (2. A.), p. 370 : «Ein Stiefeltritt, ein Knüppelstreich/ Im dritten, christlich-deutschen Reich ». Trad. A. Lerousseau pour tous les extraits du recueil, non traduit à ce jour en français.

${ }^{3}$ H. Pillau, «'Anno Domini 1933'. Zu dem Gedichtzyklus 'Das Wort der Stummen' von Gertrud Kolmar», op. cit. : «Das 'christliche Abendland' lässt seine Maske fallen! ».

${ }^{4}$ Voir à ce propos le Marbacher Magazin 63/1993: Gertrud Kolmar 1894-1943, présenté par Johanna Woltmann, p. 5.
} 
associé à ceux des autres écrivaines juives d'origine allemande qui, du fait de leur naissance ou de leur vie, sont intimement liées à la ville de Berlin. Ainsi que le remarque Thomas Sparr, fin connaisseur de l'œuvre de Kolmar :

Gertrud Kolmar n'est plus de nos jours une inconnue, elle est célébrée au même titre qu'Else Lasker-Schüler ou Nelly Sachs, avec lesquelles elle constitue une triade judéo-allemande qui resplendit dans le ciel obscur de Berlin au 20ème siècle. $^{5}$

La description qu'elle fait implicitement d'elle-même dans le poème intitulé « L'Inexplorée » [ « Die Unerschlossene »], dans le recueil Portrait de femme [Weibliches Bildnis], est éclairante. Le titre contient déjà in nuce tout le poème. Avec une concision extrême, il met en lumière le décalage qui existe entre les richesses potentielles d'un être et la façon dont il est perçu de l'extérieur. Dès le premier vers, un moi lyrique, que l'on s'imagine pouvoir cerner très vite, revendique avec insistance sa nature inépuisable: «Moi aussi, je suis une partie du monde $»^{6}$. Et le poème se termine par cette strophe :

Je suis souvent surplombée par des ciels aux astres noirs, aux orages irisés,

En moi sont des cratères effrangés, dentelés, qui tremblent sous une contrainte rougeoyante ;

Mais il y a là aussi une source pure et glacée et la campanule qui la boit :

Je suis un continent qui un jour sombrera muet dans la mer. ${ }^{7}$

Le « moi », avec sa plénitude intérieure, s'est accoutumé à l'idée de demeurer indiscernable. Ce qui l'habite ne sera pas mis en valeur, mais la conscience de son intégrité un peu revêche lui suffit. À vrai dire, aussi longtemps que la propension à se confier et à tisser des liens

\footnotetext{
${ }^{5}$ Gertrud Kolmar, Liebesgedichte, ausgewählt und mit einem Nachwort versehen von Thomas Sparr, Berlin, Insel-Verlag 2010, p. 101 : « Kolmar ist heute keine Unbekannte mehr, sowenig wie Else Lasker-Schüler oder Nelly Sachs, die ein leuchtendes deutschjüdisches Dreigestirn am dunklen 20.-Jahrhundert-Himmel über Berlin bilden ».

${ }^{6}$ G. Kolmar, Das lyrische Werk. Gedichte 1927-1937, op. cit., p. 95 : « Auch ich bin ein Weltteil ». Pour la traduction française: Gertrud Kolmar, Quand je l'aurai tout bu (Poésies 1927-1932), poésies traduites par Fernand Cambon, introduction d'Alain Lercher, Strasbourg, les éditions Circé, 2014, p. 43.

${ }^{7}$ Ibid., pour la traduction française ; p. 95-96 pour l'édition allemande : « Über mir sind oft Himmel mit schwarzen Gestirnen, bunten Gewittern,/ In mir sind lappige, zackige Krater, die von zwingendem Glühen zittern ;/ Aber auch ein eisreiner Quell und die Glockenblume ist da, die ihn trinkt :/ Ich bin ein Kontinent, der eines Tages stumm im Meere versinkt ».
} 
déboucheront sur du vide, l'inexploré permettra au moins d'espérer encore.

Le recueil intitulé La Parole des muets est sans équivalent dans l'histoire de la littérature allemande. Confronté à l'abîme qui s'ouvrait en 1933 dans l'histoire allemande, aucun poète, à l'exception de Gertrud Kolmar, n'a en effet réagi avec autant de rapidité, de sensiblitité et avec une telle force expressive et un tel réalisme. On se serait d'autant moins attendu à cela venant d'une poétesse dont les motifs de prédilection avaient jusqu'alors été les fleurs, les animaux, les blasons des villes ou la polarité des sexes. Son père lui-même, avec lequel elle vivait depuis le décès de sa mère dans une maison à la périphérie de Berlin (à «Finkenkrug », dans le district de «Falkensee »), pensait comme beaucoup d'autres qu'il ne fallait pas prendre trop au sérieux l'agitation politique des nouveaux détenteurs du pouvoir. Les événements survenus au sein de leur propre famille auraient pourtant dû les alarmer. Georg Benjamin, médecin et cousin de Gertrud Kolmar, avait été placé en « détention préventive » le 8 avril 1933 en tant que communiste, avant d'être envoyé le 4 juillet de la même année dans le camp de concentration de Sonnenburg ${ }^{8}$. Quant à Walter Benjamin, son plus jeune frère, qui devint plus tard un philosophe célèbre, il avait quitté 1'Allemagne peu de temps après la " prise de pouvoir ${ }^{9}$ d'Hitler, le 30 janvier 1933. Il séjournait depuis mars à Paris et ne devait plus jamais fouler le sol allemand ${ }^{10}$. Ces épisodes ont très probablement poussé Gertrud Kolmar à rédiger des poèmes politiquement engagés, contrairement à ses textes antérieurs.

En 1933, la poétesse semble se dire qu'il est impossible de continuer à écrire comme auparavant, ce qui aurait porté préjudice à sa poésie que l'on aurait considérée comme une échappatoire face à l'urgence de l'époque. Le devoir prioritaire d'un poète n'était-il pas aujourd'hui de prêter sa voix à ceux que la torture avait réduits au

\footnotetext{
${ }^{8}$ Voir à ce propos Uwe-Karsten Heye, Die Benjamins. Eine deutsche Familie, Berlin, Aufbau-Verlag, 2014, p. 131 et 134.

9 «Machtergreifung », terme inapproprié, Hitler étant parvenu au pouvoir par la voie légale.

${ }^{10}$ Cf. Johanna Woltmann, Gertrud Kolmar. Leben und Werk, Frankfurt a. M., Suhrkamp, 2001, p. 196.
} 
silence ? C'est en ce sens que l'on interprète généralement le titre du recueil. D'un autre côté, Gertrud Kolmar a parfaitement conscience du fait que la langue ne demeure vivante qu'à la condition de ne pas être subordonnée à des desseins certes très honorables, mais déterminés par avance, mais de se tenir prête à accueillir des contenus qui fermentent et ne sont pas réductibles à des intentions. Dans ses poèmes, la langue effectue ainsi de brusques écarts métaphoriques rappelant le surréalisme, mais qui, à la différence du lyrisme expressionniste de l'époque, demeurent prisonniers du corset de la rime. On ressent l'intensité de la ferveur qui jamais ne s'enflamme. D'où la comparaison, établie par de nombreux commentateurs, de cette poésie avec un rubis.

Face à ces défis politiques qui la touchent de près, Gertrud Kolmar éprouve un déchirement dont témoignent à la fois la place singulière qu'occupe le recueil dans son œuvre et le cycle lui-même. Seuls sept poèmes, sur les vingt-deux qu'il contient, sont une réaction à la situation politique $^{11}$. Ils sont encadrés de textes rappelant les écrits antérieurs dont la tonalité dominante est celle du deuil, de la mélancolie et d'un état d'âme empreint de religiosité. Ils tournent souvent autour du thème de l'enfant qui n'est jamais venu au monde, un motif récurrent chez Gertrud Kolmar suite à un avortement auquel elle fut contrainte dans sa jeunesse. Le deuil de l'enfant mort et la tendre invocation de sa présence sont étrangement mêlés. À cela s'ajoutent quelques poèmes que nous qualifierions de passerelles entre les textes apolitiques et politiques.

L'opposition entre un lyrisme intemporel et une poésie engagée ne se reflète pas seulement dans la conception du recueil, mais également dans des poèmes pris séparément qui deviennent pour ainsi dire le théâtre où a lieu la répétition de la scène du déchirement : on mentionnera ici «Die gelbe Rose» « La rose jaune »] et «Die Gefangenen » [ Les prisonniers»]. Le premier poème semble d'emblée s'inscrire dans le prolongement d'un cycle antérieur rassemblant des sonnets consacrés à différentes espèces de roses ${ }^{12}$. Les premières strophes sont une méditation sur la rencontre entre un être humain et la rose. Tandis que le moi lyrique se confie à la rose, celle-ci s'ouvre également à lui. Échappant désormais aux contraintes liées à son identité, ce dernier est accueilli dans un espace de paix et d'humanité (« La rose jaune [..] se

\footnotetext{
${ }^{11}$ Sur la composition du cycle, voir Ingeborg Fiala-Fürst, «Der Gedichtzyklus 'Das Wort der Stummen' ", dans Widerstehen im Wort. Studien zu den Dichtungen Gertrud Kolmars, hrsg. von Karin Lorenz-Lindemann, Göttingen, Wallstein-Verlag, 1996, p. 35. ${ }^{12}$ G. Kolmar, Bild der Rose. Ein Beet Sonette, dans Das lyrische Werk, Gedichte 19271937, op. cit., p. 323-343.
} 
répand en parfum [...] À travers la pièce [...] // Épouse docilement mes formes sans demander qui je suis, / D'où a jailli mon sang rouge sous la chevelure sombre $»)^{13}$ et se berce même de l'idée que les lois du temps pourraient être abolies sous la douce domination de la fleur qui « [...] a peut-être échappé à la couronne de la mort éternelle $»{ }^{14}$.

Le lecteur est comme abasourdi quand, dans la dernière strophe, cette sérénité est détruite par l'irruption soudaine de la réalité. L'expérience d'une utopie humaine est balayée par une "dérision sacrée ${ }^{15}$. Des seigneurs dominent la scène de leur présence animale et tentent de s'élever en rabaissant les autres :

Et qu'ils hennissent ou trépignent comme de fiers chevaux d'élevage

L'homme du Nord s'entend mieux à tirer gloire que le Juif ou l'Hottentot

Et le prêtre dans un ciel exigu lui procure un nouveau dieu $-{ }^{16}$

La rose jaune cède brusquement la place à des hordes de SA qui défilent dans la rue en beuglant, et les nouveaux maîtres ne se contentent pas de terroriser les gens, ils ont également la velléité de mêler la transcendance à leurs desseins. Le ciel est « exigu », car il ne sert que les intérêts du pays. Gertrud Kolmar semble ici faire une nouvelle allusion à la tentative des nazis d'instrumentaliser l'Église protestante à leur profit par le biais des « Chrétiens allemands » ${ }^{17}$.

Tandis que dans « La rose jaune » le lecteur est induit en erreur par le titre, les choses paraissent beaucoup plus claires dans «Les prisonniers ». Contrairement toutefois à ce que laissaient présager le titre du poème et la mise en exergue de l'année 1933, les deux premières strophes évoquent avec tendresse l'époque des récoltes en automne. Cette saison nous enchante avec son « grand panier rempli de tomates dodues,/

\footnotetext{
${ }^{13}$ Ibid., p. 360 : «Die gelbe Rose [...] zieht in Duft [...] Durch meine Stube [...]// Umschmiegt mich willig und achtet nicht, wer ich bin,/ Woher unter finsteres Haar mein rotes Blut gesprungen ».

${ }^{14}$ Ibid., « Und ist, mag sein, dem Kranz des ewigen Todes entfallen, [...]».

${ }^{15}$ Ibid., « heiligen Spott».

16 Ibid., «Und ob sie wiehern und stampfen wie stolz gezüchtete Pferde,/ Der Nordmann besser sich preist als Jude und Hottentott/ Und der Priester im engen Himmel ihm schafft einen neuen Gott - ».

${ }^{17}$ Aujourd'hui encore, on observe des tendances à donner une coloration nationaliste au christianisme, ainsi par exemple au sein du nouveau parti, l'AfD (Alternative für Deutschland). D'où la remarque du théologien Gregor-Maria Hoff : « Le Dieu de Jésus Christ devient un dieu national». "So wird aus dem Gott Jesu Christi ein Nationagott», dans «Gott wird zum Nationalgott. Über das unchristliche Bekenntnis der AfD », Die Zeit, 14. 9. 2017, p. 54.
} 
Empli de prunes bleutées, mêlées à la pomme et à la poire jaune ${ }^{18}$. Un garçon lance son cerf-volant, une jeune femme et un « vieux qui souffre de la goutte» [ « ein gichtiger Alter»] s'arment contre le froid qui s'insinue lentement ${ }^{19}$. Dans la troisième strophe cependant, des ombres planent sur cette idylle : des mendiants sonnent à la porte, un « invalide de guerre » [《Kriegskrüppel», «Krüppel» désignant un cul-de-jatte] fait son apparition, il est fait mention de la «mort effroyable» de «malheureux amants» et des peines éprouvées par des " petites femmes ratatinées» [«hutzelnde Weibchen»] pour brader leurs misérables marchandises ${ }^{20}$. L'ambiance idyllique se dissipe totalement lorsque l'on est confronté à la condition des prisonniers dans un camp. Ils ne sont pas traités comme des êtres humains, pas même comme des êtres vivants. D'où leur sentiment : «Et selon toute vraisemblance, ils sont encore en vie. Cela, ils ne peuvent le concevoir $»^{21}$. Leur existence se résume à l'attente de la mort : « Depuis longtemps traînés à l'abattoir, ils attendent le couteau, muets $\gg{ }^{22}$. Sans doute le poème ne débute-t-il par la description de scènes automnales idylliques que pour mieux souligner le contraste avec l'horreur du camp.

Le lien étroit entre les poèmes politiques de Gertrud Kolmar contenus dans le cycle et les événements ressort clairement dans le poème «An die Gefangenen » [«Aux prisonniers »]. La dédicace - ou ce qui en tient lieu - est formulée ainsi : «À l'occasion de la fête d'action de grâces pour la récolte du $1^{\text {er }}$ octobre $1933 »^{23}$. La poétesse rédigea ce texte la veille, le 30 septembre $^{24}$. Si Hitler érigea cette fête en manifestation officielle ${ }^{25}$, il s'efforça de l'infléchir dans le sens du national-socialisme. Elle devait éveiller chez les masses qui y assistaient le sentiment de constituer une «communauté du peuple » [«Volksgemeinschaft»]. À l'inverse, ceux qui à l'instar des prisonniers n'avaient pas le droit d'y participer étaient exclus de cette communauté.

Gertrud Kolmar déploie toute sa ferveur lyrique afin d'arracher les prisonniers à leur isolement. Grâce à cet élan extatique, ceux-ci seraient

\footnotetext{
${ }^{18}$ G. Kolmar, Das lyrische Werk. Gedichte 1927-1937, op. cit., p. 363 : « einen großen Korb voll praller Tomaten, Voll blauer Pflaumen, mit Apfel und gelber Birne ».

${ }^{19}$ Ibid.

${ }^{20}$ Ibid.

${ }^{21}$ Ibid., « Und leben wahrscheinlich noch. Das können sie nicht begreifen ».

${ }^{22}$ Ibid., « Längst in den Schlachthof getrieben, warten sie stumm auf das Messer ».

${ }^{23}$ Ibid., p. 365 : «Zum Erntedankfest am 1. Oktober $1933 »$.

${ }^{24}$ G. Kolmar, Das lyrische Werk, Anhang und Kommentar, op. cit., p. 263.

${ }^{25}$ En particulier lors d'une mise en scène sur le Bückeberg, aux environs de Hameln (cf. ibid.).
} 
en mesure de se percevoir de l'extérieur : «[...] - Ô Dieu, nous les hommes sommes des nains $»^{26}$. Mais la poétesse est également consciente du fait que sur le fond rien ne la différencie des prisonniers, ce qui la conduit à se représenter son destin à venir :

Cela adviendra, oui, cela adviendra ; surtout, ne vous méprenez !

Car quand ils trouveront ces lignes, de moi ils se saisiront.

Seigneur, fasse que, les yeux ouverts, je comparaisse devant ton tribunal sacré, Le jour où par ma chevelure ensanglantée à travers de sombres ouvertures ils me traîneront $!^{27}$

Ces vers sont terribles, en ce qu'ils font de prime abord l'effet d'une capitulation de la part de la poétesse : à l'instant même où elle parviendrait à proximité des prisonniers et où ses mots pourraient enfin les atteindre, elle aussi y laisserait la vie. Son triomphe poétique est concomitant de sa perte. Mais ces vers nous plongent dans l'effroi également pour une autre raison : le destin du moi lyrique qui est invoqué ici correspond en effet, comme nous le savons, à celui qui sera le sien.

Elle confie par ailleurs que, de la douleur qui menace de lui ôter la parole, pourrait jaillir le chant, la poésie :

Misérable, blessée, je veux donner libre cours à mon désespoir, à mes larmes, Et chanter semblable à l'oiseau dont l'œil est transpercé d'aiguilles $!^{28}$

Quand nous lisons ce poème, ainsi que d'autres textes du recueil comme «Ewiger Jude» [ « Le Juif éternel»] et «Der Misshandelte » [«L'homme victime de sévices»], nous sommes frappés par la clairvoyance de Gertrud Kolmar. En effet, il ne faut pas oublier que l'on ne pouvait prévoir en 1933 l'ampleur qu'allait prendre la persécution des Juifs. Pour nombre de Juifs allemands, comme pour son père, la référence demeurait l'État de droit de la République de Weimar avec ses normes. Gertrud Kolmar sentait toutefois la nécessité qu'il y avait à arracher les Juifs au délire d'une intégration sociale, ainsi qu'en témoigne

\footnotetext{
${ }^{26}$ G. Kolmar, Das lyrische Werk. Gedichte 1927-1937, op. cit., p. 365 : « [...] - O Gott, wir Menschen sind Zwerge ».

${ }^{27}$ Ibid., « Das wird kommen, ja, das wird kommen ; irret euch nicht!/ Denn da dieses Blatt sie finden, werden sie mich ergreifen./ Herr, gib, daß ich wach mich stelle deinem heiligen großen Gericht,/ Dann, wenn sie an blutendem Schopf durch die finsteren Löcher mich schleifen! ». (Note de la traductrice : nous avons tenté, de façon plus ou moins heureuse, de maintenir la rime).

${ }^{28}$ Ibid., p. 366 : «Verzweifeln will ich, will aufweinen, elend, verletzt,/ Und singen dem Vogel gleich, dem Nadeln das Auge stechen!».
} 
le poème « Wir Juden » [ « Nous les Juifs »] visant à éveiller une nouvelle conscience juive de soi. Pour ce faire, elle recommande une étonnante stratégie: au lieu de poursuivre leurs efforts d'assimilation ou, à l'inverse, de se rebeller, les Juifs doivent plutôt accepter leur mise à l'écart. Consentir à ce que fait de nous un environnement hostile, revient à perturber ce jeu malveillant et démoralisant. Les Juifs ne perçoivent plus alors leur différence comme une tare, à l'instar de leur entourage, mais au contraire comme une qualité qui leur est inhérente. Ils posent alors un regard neuf sur eux-mêmes et sur le monde environnant. Cela se traduit par exemple par le fait qu'ils sont immunisés contre les tentations du pouvoir et contre l'opportunisme :

Je ne baiserai pas le bras aux muscles saillants, agitant un sceptre orgueilleux, Ni le genou d'airain, ni le pied d'argile de l'idole d'un temps cruel. ${ }^{29}$

Mais ce qui est plus important est la perception qu'ont les Juifs d'une société humaine, soucieuse de la participation de tous aux biens de la vie, dimension qui s'estompe dès que les rapports de pouvoir s'autonomisent. Gertrud Kolmar songe ici à la justice dont les Juifs pressentent la disparition à leurs propres dépens :

Si seulement, flambeau ardent dans le désert sombre du monde, je pouvais Élever ma voix : Justice ! Justice ! Justice $!^{30}$

Avec des accents quasi révolutionnaires, elle entame ici un combat afin de les extraire de ce cul-de-sac que représente l'assimilation.

Dans le poème « Die jüdische Mutter » [ « La mère juive »], la mère s'adresse directement aux non-Juifs. Elle tente d'illustrer ce que la discrimination quotidienne provoque chez son enfant, tout en attirant l'attention sur la dénaturation que s'infligent à eux-mêmes les petits sadiques par leurs agissements: "L'envie, la méchanceté, la lâche frénésie, ce qui profane votre visage, $/[\ldots] »^{31}$. Nous sommes de nouveau confrontés au fossé qui sépare une culture prétendument chrétienne de la pratique :

\footnotetext{
${ }^{29}$ Ibid., p. 372 : « Ich will den Arm nicht küssen, den ein strotzendes Zepter schwellt,/ Nicht das erzene Knie, den tönernen Fuß des Abgotts harter Zeit ».

${ }^{30}$ Ibid., «O könnt` ich wie die lodernde Fackel in die finstere Wüste der Welt/ Meine Stimme heben : Gerechtigkeit! Gerechtigkeit ! Gerechtigkeit !».

${ }^{31}$ Ibid., p. 373 : « Neid, Bosheit, feige Wut, was euer Anlitz schändet, / [...]».
} 
Croyez-vous qu'il soit juste, dans les églises de prier en chœur,

D'acquiescer avec satisfaction à ce que dit le pasteur,

Et en sortant de piétiner cette âme comme un animal?

Ah, celui qui a quelques égards n'écrase pas même l'animal ! ${ }^{32}$

Bien que la mère sente monter en elle la fureur, elle ne voudrait pas se laisser subjuguer par ce sentiment, ce qui la mettrait au même niveau que les agresseurs. Au lieu de cela, elle mise sur la capacité de résistance de son enfant dans l'avenir avec l'espoir que celui-ci ne s'endurcira pas : «Lève-toi, mon enfant, et accuse avec tes jeunes paroles $!-{ }^{33}$.

Considérons pour finir le poème « Der Engel im Walde » [ «L'ange dans la forêt »] qui clôt le recueil. En se laissant aller à la révolte, à la protestation et à l'agitation en ces temps de détresse, la langue poétique risquait de s'appauvrir ${ }^{34}$. Le poème fait ainsi l'effet d'un rappel des possibilités inhérentes à cette langue. Le moi lyrique se sent attiré par l'ange dans la forêt qui en même temps le déçoit : en effet, il reste muet, replié sur lui-même et «éloigné des hommes » [«menschenfern »] $]^{35}$. Seuls, l'écureuil et les chevreuils le comprennent. Lorsque l'homme exige quelque chose de lui, il se dérobe. Si au contraire l'homme le laisse tranquille, il agit par sa présence silencieuse. Le cycle se termine sur cet aveu de la nature paradoxale de la poésie.

Le recueil témoigne de la condition à laquelle la pression énorme exercée par les événements contraint Gertrud Kolmar en tant que poète. À travers son lyrisme politique, elle parvient certes à trouver le mot ardemment recherché afin de traduire ces temps de détresse, mais semble

\footnotetext{
${ }^{32}$ Ibid., " Glaubt ihr, es sei gerecht, in Kirchen mitzubeten,/ Behaglich anzunehmen, was der Pfarrer spricht,/ Dann hinzugehn und diese Seele wie ein Tier zu treten?/ Ach, auch das Tier zertritt der Wohlbedachte nicht! ». (Note de la traductrice : nous nous nous sommes efforcée de rendre les rimes, bien que selon un schéma différent).

${ }^{33}$ Ibid., p. 374 : «Steh' auf, mein Kind, und klage an mit deinem jungen Wort ! - ».

${ }^{34}$ Cf. I. Fiala-Fürst, «Der Gedichtzyklus, Das Wort der Stummen' », op. cit., p. 43. Dans un registre plus général, voir la remarque du poète américain Matthew Zapruder : «Sie [die Poesie] dient keinem anderen Zweck, nicht der Narration, der Argumentation, dem An- und Verkauf, der Predigt und der Verurteilung ». [«Elle [la poésie] ne sert aucun autre dessein, ni la narration, ni l'argumentation, ni les achats, ni les ventes, ni les sermons, ni la condamnation »], dans Matthew Zapruder : «Zimmer mit Aussicht. Was vermag die Poesie in Zeiten der Krise? Gedichte sind Orte der Begegnung, der Freiheit », dans Der Tagespiegel, 8. 10. 2017. http://www.tagespiegel.de/kultur/essayzu-poesie- und-politik-zi...

${ }^{35}$ G. Kolmar, Das lyrische Werk. Gedichte 1927-1937, op. cit., p. 385.
} 
par là-même renoncer à une langue poétique indépendante de l'actualité et n'obéissant qu'à ses propres règles. Ainsi que l'indiquent plusieurs remarques dans ses lettres à sa sœur Hilde, elle revendiquera plutôt les années suivantes une poésie atemporelle dans laquelle il sera question non pas de l'actualité, mais de «ce qui a lieu dans l'éternité" [«Ewigkeitsgeschehen»] $]^{36}$. Elle se concentre sur «ce qui demeure, animaux et plantes, ce qui ne cesse de revenir, ce qui persiste à travers l'impermanence et le changement $»^{37}$. C'est sous cet éclairage qu'elle perçoit ses premiers écrits : «Et de ma propre expérience, je sais que je prends bien plus rarement comme objet de ma poésie ce qui est proche, actuel, que ce qui est passé ${ }^{38}{ }^{3}$. Le fait que, dans ses poèmes, elle se détourne du présent pour se retirer dans l'immuable, pourrait bien être lié à sa perception de son propre présent. En tant que Juive, elle fait immanquablement l'expérience du rétrécissement de son espace vital suite aux chicanes toujours plus sophistiquées imaginées par les services administratifs. La conséquence pour elle est le relâchement progressif du rapport qu'elle entretient intérieurement au présent immédiat, ne fût-ce que pour se protéger. Elle confie à sa soeur dans sa lettre du 2 juin 1941 :

Moi au contraire, je ne me sens de proximité qu'avec le passé, c'est ce qui m'arrive aujourd'hui qui est irréel et lointain pour moi. Si je ne rêve pas vraiment, je ne suis pas éveillée pour autant. Je me promène comme dans un monde intermédiaire qui n'a aucune part en moi et auquel je n'ai aucune part. ${ }^{39}$

L'interruption de tout contact vivant avec le présent aurait pu entraîner dans sa poésie une sorte d'engourdissement dans une sphère hors du temps, et finalement un mutisme. Son dernier recueil, Welten [Mondes], prouve que ce ne fut pas le cas. Ce cycle, qui vit le jour entre octobre et décembre 1937, est unanimement considéré comme son chef $\mathrm{d}^{\prime} œ u v \mathrm{ure}^{40}$. De plus en plus rejetée hors de son propre monde, elle gagne

\footnotetext{
${ }^{36}$ Gertrud Kolmar, Lettres, trad. de l'allemand par Jean Torrent, Paris, Christian Bourgois Éditeur, 2001, p. 69. Dans sa lettre datée du 18 novembre 1942, Gertrud Kolmar écrit qu'elle s'efforce d'appliquer «à toute chose, à tous les événements la mesure de l'éternité » (ibid., p. 266). Pour les lettres citées, le lecteur germanophone pourra se reporter à l'édition allemande: Gertrud Kolmar, Briefe, hrsg. von Johanna Woltmann, Göttingen, Wallstein-Verlag, 2014.

${ }^{37}$ G. Kolmar, Lettres, op. cit., p. 70 (lettre du $1^{\text {er }}$ octobre 1939).

${ }^{38}$ Ibid., p. 121 (lettre du 14 juillet 1940).

${ }^{39}$ Ibid., p. 163-164.

${ }^{40}$ Cf. G. Kolmar, Das lyrische Werk. Gedichte 1927-1937, op. cit., p. 503- 545. Pour la traduction française : Gertrud Kolmar, Mondes, édition établie, postfacée et traduite de l'allemand par Jacques Lajarrige, Paris, Seghers, 2001.
} 
cette liberté intérieure de créer en imagination des mondes alternatifs. Ceux-ci, par leur plénitude de vie et leur fécondité, surpassent de très loin le monde réel devenu inhabitable. Pour la première fois, elle utilise des vers libres. Après quoi Gertrud Kolmar se tait en tant que poétesse allemande - mais non en tant que narratrice ${ }^{41}$. Possédant un diplôme d'interprète, elle maîtrise parfaitement l'anglais et le français et se met à l'apprentissage de l'hébreu, langue dans laquelle elle écrira des poèmes qui hélas ont été perdus ${ }^{42}$.

La soi-disant « Nuit de Cristal» du 9 novembre 1938 ne fut pas sans conséquence pour la vie de Gertrud Kolmar et de son père. Celui-ci, âgé de 77 ans, fut incarcéré et libéré au bout de quatre jours. Peu de temps après, il fut contraint de vendre sa villa de Finkenkrug à la périphérie de Berlin pour un prix dérisoire. Rétrospectivement, cette maison entourée d'un grand jardin apparaissait à Gertrud Kolmar, amie de la nature, comme un "paradis perdu " ${ }^{43}$. Le père et la fille durent déménager à Schöneberg, un quartier situé dans le centre de Berlin, où on leur attribua un grand appartement, qui se peupla toutefois peu à peu d'autres Juifs berlinois assignés là à résidence. Si les deux sœurs de Gertrud Kolmar parvinrent à émigrer, elle ne quitta pour sa part pas l'Allemagne, soucieuse avant tout, selon son propre aveu, de demeurer auprès de son père âgé et de santé fragile ${ }^{44}$. Il fut déporté en 1942, à 82 ans, dans le camp de concentration de Theresienstadt où il mourut peu de temps après. Son courrier à destination de l'étranger étant censuré, Gertrud Kolmar ne pouvait relater ces événements à sa sœur que dans une langue codée. Condamnée depuis juillet 1941 au travail forcé dans des usines d'armement, elle fut emprisonnée le 13 février 1943 dans le cadre d'une soi-disant «Fabrikaktion» [intervention dans les usines] et déportée à Auschwitz où elle fut vraisemblablement directement envoyée dans la chambre à gaz.

\footnotetext{
${ }^{41}$ Entre le 29 décembre 1939 et le 13 février 1940, elle rédige le récit Susanna: Gertrud Kolmar, Susanna. Erzählung, mit einem Nachwort von Thomas Sparr, Frankfurt a. M., Suhrkamp Verlag, 1995. Pour la traduction française : Gertrud Kolmar, Susanna, trad. de Laure Bernardi, Paris, Bourgois (coll. Titre 41), 2007.

${ }^{42}$ Voir à ce propos G. Kolmar, Lettres, op. cit., p. 114-115 (lettre du 15 mai 1940).

${ }^{43}$ Cf. Dieter Kühn, Gertrud Kolmar. Leben und Werk, Zeit und Tod, Fischer, Frankfurt a. M., 2010, p. 423.

${ }^{44}$ G. Kolmar, Lettres, op. cit., p. 68 (lettre du 10 septembre 1939).
} 
(7)

Le manuscrit du recueil La Parole des muets a été conservé, Gertrud Kolmar l'ayant confié peu après la déportation de son père à Hilde Benjamin, la femme de son cousin Georg, incarcéré dans le camp de concentration de Mauthausen où il fut assassiné en 1941. Hilde Benjamin enterra le manuscrit et les lettres de son mari dans son jardin ouvrier. Elle extirpa ces documents en 1946, craignant toutefois, selon son propre témoignage, de les faire connaître. Qu'elle soit devenue plus tard, de 1953 à 1967, une ministre de la justice particulièrement stricte et de ce fait très controversée en RDA, s'explique sans doute par ce qu'elle avait vécu sous le Troisième Reich. Ce n'est qu'à la fin des années soixante-dix qu'elle s'occupa de la publication du recueil. Le livre paru en 1978 contient, outre les poèmes et la postface d'un poète renommé, un texte dans lequel elle évoque ses souvenirs de Gertrud Kolmar ${ }^{45}$. Dans la RDA qui se définissait essentiellement par l'antifascisme, l'œuvre de celle-ci faisait partie du patrimoine culturel national. Dans la République fédérale par contre, on ne s'intéressa dans un premier temps qu'à ses écrits apolitiques, avant tout donc à sa poésie de la nature. Concernant d'autres textes plus explosifs - nous pensons ici en particulier à ses écrits sur Robespierre ${ }^{46}$ - on eut tendance à les contourner.

\footnotetext{
${ }^{45}$ Gertrud Kolmar, Das Wort der Stummen. Nachgelassene Gedichte, hrsg. und mit einem Nachwort von Uwe Berger und «Erinnerungen an Gertrud Kolmar» von Hilde Benjamin, Berlin, Verlag der Morgen, 1978.

${ }^{46}$ Voir entre autres le cycle Robespierre, dans G. Kolmar, Das lyrische Werk. Gedichte 1927-1937, op. cit., p. 391-475, et l'écrit en prose, Das Bildnis Robespierres, dans G. Kolmar, Das lyrische Werk, Anhang und Kommentar, op. cit., p. 19-52. Pour l'édition française: Gertrud Kolmar, Robespierre et Le Portrait de Robespierre, traduction de l'allemand et postface de Sibylle Müller, Strasbourg, les éditions Circé, 2017.
} 